\title{
Efficacy of primary tumour volume as a predictor of survival compared with size alone in pancreatic ductal adenocarcinoma
}

\author{
JUSTIN S. GUNDARA ${ }^{1 *}$, ANTHONY J. GILL $^{2 *}$ and JASWINDER S. SAMRA ${ }^{1 *}$ \\ ${ }^{1}$ Upper Gastrointestinal Surgical Unit; ${ }^{2}$ Department of Anatomical Pathology, Royal North Shore Hospital, \\ University of Sydney, St. Leonards, NSW 2065, Australia
}

Received August 12, 2014; Accepted April 14, 2015

DOI: $10.3892 / \mathrm{ol} .2015 .3254$

\begin{abstract}
Tumour size (TSize) predicts outcome in pancreatic ductal adenocarcinoma (PDAC), but little is known regarding three-dimensional tumour volume (TVol) associations. We hypothesised that TVol would more accurately predict outcome following pancreatoduodenectomy (PD) for PDAC. Clinicopathological and outcome data was reviewed for all PDs performed in the Royal North Shore Hospital (St. Leonards, NSW, Australia), between April 2004 and November 2010, in patients whose three tumour dimensions were recorded $(n=103)$. TVol was quantified using the ellipsoidal volume formula, $4 / 3 \pi(\mathrm{r} 1 \times \mathrm{r} 2 \times \mathrm{r} 3)$, and was correlated with clinicopathological indices/outcome. Over a median follow-up time of 20.5 months, TVol failed to significantly predict post-resection mortality [odds ratio (OR), 1.0; 95\% confidence interval (CI), 0.99-1.00; $\mathrm{P}=0.438)$. Neural invasion remained an overall independent predictor of mortality following multivariate analysis (OR, 3.94; 95\% CI, 1.36-11.40; $\mathrm{P}=0.011$ ). Patients with higher $\mathrm{TV}$ ol were more likely to require a vascular resection $(\mathrm{P}=0.007)$, had longer surgical times $(\mathrm{P}<0.001)$, larger intraoperative blood losses $(\mathrm{P}=0.007)$ and a trend toward worse survival $(\mathrm{P}=0.068)$. TVol inclusion in a multivariate model resulted in a small improvement in mortality prediction versus TSize (14.9 vs. 14.7\%). A higher TVol results in a more complex perioperative course. Although TVol improved the mortality prediction beyond simple TSize alone, this difference was not significant. Studies normalising TVol for body composition are required.
\end{abstract}

\section{Introduction}

Tumour size (TSize) has long been used as a variable of prognostic significance and is employed universally in

Correspondence to: Professor Jaswinder S. Samra, Upper Gastrointestinal Surgical Unit, Level 8, Royal North Shore Hospital, University of Sydney, Pacific Highway, St. Leonards, NSW 2065, Australia

E-mail: jaswinder.samra@optusnet.com.au

*Contributed equally

Key words: pancreatic ductal adenocarcinoma, tumour volume staging systems for all solid tumours (1). With the evolution of surgical techniques, boundaries continue to be pushed and the prediction of which patients will definitively benefit from resection has never been so important. Accordingly, there is an increased emphasis on improving staging systems that incorporate variables of the greatest prognostic significance. In pancreatic ductal adenocarcinoma (PDAC), primary tumour characteristics are known to be the most important determinants of outcome (2).

Tumour volume (TVol) has been investigated as a marker of prognosis in lung cancer (3), prostate cancer (4), renal cell carcinoma (5), tongue cancer (6), peri-ampullary malignancy (7) and osteosarcoma (8). When compared with existing staging variables, TVol has been shown to enhance outcome prediction significantly, above and beyond single dimensions such as TSize (3-6,8). This is logical, given that solid tumours are three-dimensional structures and one dimension, whilst generally associated with outcome, may not accurately reflect the primary tumour burden of the disease.

Despite the investigation of a number of solid organ tumour types, PDAC TVol has not been examined and compared with TSize specifically with regard to perioperative variables and outcome. Using a retrospective analysis of the prospective institutional database for the Royal North Shore Hospital (St. Leonards, NSW, Australia), we hypothesised that TVol would significantly predict outcome better that TSize alone, following pancreatoduodenectomy (PD) for pancreatic head PDAC.

\section{Materials and methods}

Study approval. Approval was granted from the Northern Sydney Area Health Service Human Research Ethics Committee prior to study commencement.

Patients. Pancreatic resection data from the Royal North Shore Hospital Campus of Sydney University was prospectively collated over the period of April 2004 to November 2010. A total of 198 PD's were performed during this period for a number of indications. Cases not involving PDAC as the primary indication for resection were excluded. In order to calculate $\mathrm{TV}$ ol, only cases where histopathology reports included three measured tumour dimensions were included. 
Following exclusion of non-PDAC cases and those with inadequate pathology data, 103 cases of were available for analysis.

Demographic data was obtained in addition to American Society of Anaesthesiologists (ASA) grading. Perioperative clinical data included surgical time, intraoperative blood loss, perioperative blood transfusion requirements, hospital stay (days), complications (and complication grade), in hospital and 90 day mortality. Histopathological data included three tumour dimensions, margin (R) status, evidence of neurovascular invasion and the number of lymph nodes resected (including the number positive for disease). Clinical follow up at three-month intervals (up to a year), six-month intervals (up to two years) and annually thereafter, enabled collation of survival data.

Collation of three tumour dimensions enabled analysis of primary TSize (defined by the maximum tumour dimension), mean TSize (the mean of three tumour dimensions) and TVol. TVol was calculated according to the formula for an ellipse using the radius ( $r$ ) of three reported tumour dimensions as follows: $4 / 3 \pi(\mathrm{r} 1 \times \mathrm{x} 2 \mathrm{xr} 3)(5,7,8)$. For the purposes of sub-group analysis, patients were dichotomised into high and low TVol groups (based on TVol above and below the overall median TVol, respectively). This was repeated for high and low TSize (greatest tumour dimension) to enable a comparison between TVol and TSize.

Statistical analysis. Univariate and multivariate logistic regression was used to identify $\mathrm{TVol}$ associations with outcome. $\chi^{2}$ tests, Fisher's exact tests and Mann-Whitney U tests were used to compare variables between high and low TVol groups where appropriate. Survival data was assessed between these two groups with Kaplan-Meier curves and a log-rank test. $\mathrm{P}<0.05$ was considered to indicate a statistically significant difference. All statistical analyses were performed using Stata/IC12 (StataCorp LP, College Station, TX, USA).

\section{Results}

In total 103 patients underwent PD for PDAC and had three reported macroscopic tumour dimensions available for analysis. Within this cohort, the median age was 67 years (range, $34-83$ years) and $55 \%$ of patients were male (57 males vs. 46 females). The median ASA grading was 2 (range, 1-4).

Table I shows a summary of selective clinicopathological indices for all patients. Tables II and III present univariate and multivariate regression analysis data using mortality as the dependent variable of interest.

Univariate analysis identified perioperative blood transfusion, and neural and vascular invasion to be significantly associated with post-resection mortality. When included in a multivariate regression model, neural invasion remained as the only variable significantly associated with mortality [odds ratio, 3.94; 95\% confidence interval $(\mathrm{CI}), 1.36-11.40 ; \mathrm{P}=0.011]$.

Pairwise analyses showed significant correlations between TSize and the variables of mean TSize (coeff., 0.87; $\mathrm{P}<0.001$ ) and TVol (coeff., 0.73; $\mathrm{P}<0.001)$. Further analysis with an emphasis on TSize and TVol revealed a modest increase in the variability accounted for by the regression model when
Table I. Clinicopathological data for all cases $(n=103)$.

\begin{tabular}{|c|c|}
\hline Variable & Value \\
\hline Median age (range), years & $66(34-83)$ \\
\hline \multicolumn{2}{|l|}{ Gender, n (\%) } \\
\hline Male & $57(55.3)$ \\
\hline Female & $46(44.7)$ \\
\hline Median ASA grade (range) & $2(1-4)$ \\
\hline Surgical time ${ }^{a}$, min & $432.9 \pm 39.7$ \\
\hline Vascular resection, $\%$ & 52.4 \\
\hline Intraoperative blood loss ${ }^{\mathrm{a}}, \mathrm{ml}$ & $660.3 \pm 63.7$ \\
\hline Perioperative transfusion, $\%$ & 22.0 \\
\hline Complications, $\%$ & 34.6 \\
\hline Hospital stay, days ${ }^{a}$ & $15.8 \pm 1.3$ \\
\hline $\mathrm{TSize}^{\mathrm{a}, \mathrm{b}}, \mathrm{mm}$ & $38.5 \pm 3.4$ \\
\hline Mean TSize ${ }^{\mathrm{a}, \mathrm{c}}, \mathrm{mm}$ & $30.1 \pm 1.1$ \\
\hline Tumour volume ${ }^{\mathrm{a}}, \mathrm{cm}^{3}$ & $19.7 \pm 1.9$ \\
\hline \multicolumn{2}{|l|}{ Histological grade ${ }^{\mathrm{d}}, \mathrm{n}(\%)$} \\
\hline Well & $4(3.9)$ \\
\hline Moderate & $68(66.0)$ \\
\hline Poor & $27(26.2)$ \\
\hline Undifferentiated & $3(2.9)$ \\
\hline Neural invasion, $\%$ & 73.8 \\
\hline Vascular invasion, $\%$ & 67.9 \\
\hline Positive margin, $\%$ & 34.9 \\
\hline Lymph node yield ${ }^{a}$ & $19 \pm 1.7$ \\
\hline Lymph node-positive, $\%$ & 70.8 \\
\hline Overall 2-year survival, $\%$ & 34.1 \\
\hline
\end{tabular}

ASA, American Society of Anaesthesiologists; SE, standard error; TSize, tumour size. ${ }^{\mathrm{a} M e a n} \pm$ standard error; ${ }^{\mathrm{b}} \mathrm{Max}$. dimension; ${ }^{\mathrm{c}}$ Mean of three dimensions; degree of differentiation.

including TVol (without TVol, 14.5\%; $\mathrm{P}=0.002$; vs. with TVol, 15.7\%; $\mathrm{P}=0.001)$. When specifically comparing TVol with TSize (by exchanging them in the regression analysis), TVol marginally improved the prediction of mortality $(14.7 \%$, $\mathrm{P}=0.001$ vs. $14.9 \%, \mathrm{P}=0.001)$. Additionally, linear regression analysis demonstrated a significant negative correlation between the pancreatic neck margin and TVol $(\mathrm{P}=0.007)$.

Table IV compares outcome variables between high and low TVol cases. When compared with low TVol, high TVol cases involved longer surgical times $(\mathrm{P}<0.001)$, a greater requirement for vascular resection $(\mathrm{P}=0.007)$ and higher intraoperative blood losses $(\mathrm{P}=0.007)$.

The two-year survival rate was $41.2 \%$ in the low TVol group compared with $26.9 \%$ in the high TVol group. Fig. 1 displays Kaplan-Meier survival curves between the groups [hazards ratio (HR), 1.65; 95\% CI, 0.96-2.84; $\mathrm{P}=0.068$ ). This compares with TSize, which when divided into groups above and below the median, did not approach a significant difference (HR, 1.44; 95\% CI, 0.84-2.47; $\mathrm{P}=0.182$ ). 
Table II. Univariate analysis of clinicopathological variable associations with mortality.

\begin{tabular}{llll}
\hline Variable & OR & $95 \%$ CI & P-value \\
\hline Age & 1.02 & $0.98-1.07$ & 0.302 \\
ASA & 1.20 & $0.72-2.00$ & 0.488 \\
Surgical time & 0.99 & $0.99-1.00$ & 0.619 \\
Vascular resection & 1.64 & $0.75-3.59$ & 0.212 \\
Intraoperative blood loss & 1.00 & $0.99-1.00$ & 0.159 \\
Perioperative transfusion & 4.18 & $1.42-12.37$ & 0.010 \\
Complications & 0.81 & $0.36-1.83$ & 0.613 \\
Hospital stay & 0.99 & $0.94-1.05$ & 0.785 \\
Tumour size & 1.01 & $0.99-1.04$ & 0.300 \\
Tumour volume & 1.00 & $0.99-1.00$ & 0.478 \\
Histological grade & 1.58 & $0.80-3.11$ & 0.183 \\
Neural invasion & 4.90 & $1.84-13.03$ & 0.001 \\
Vascular invasion & 3.38 & $1.41-8.09$ & 0.006 \\
Positive margin & 1.94 & $0.84-4.45$ & 0.120 \\
Lymph node-positive & 2.15 & $0.90-5.11$ & 0.083
\end{tabular}

ASA, American Society of Anaesthesiologists; CI, confidence interval; OR, odds ratio.

Table III. Multivariate analysis of clinicopathological variable associations with mortality (using a univariate P-value of $<0.1$ for model inclusion).

\begin{tabular}{llll}
\hline Variable & OR & $95 \%$ CI & P-value \\
\hline Perioperative transfusion & 2.98 & $0.95-9.35$ & 0.061 \\
Tumour size & 0.99 & $0.96-1.04$ & 0.950 \\
Tumour volume & 1.00 & $0.99-1.01$ & 0.586 \\
Neural invasion & 3.94 & $1.36-11.40$ & 0.011 \\
Vascular invasion & 2.23 & $0.79-6.30$ & 0.132 \\
Lymph node-positive & 1.24 & $0.44-3.53$ & 0.680 \\
\hline
\end{tabular}

CI, confidence interval; OR, odds ratio.

\section{Discussion}

It is well known that TSize impacts directly on clinical outcome in a multitude of cancer types, including PDAC (2), and it is a logical assumption to suggest that this would generally hold true of TVol. In keeping with this, the present analysis showed that TSize and TVol are negatively associated with survival. The real issue under contention, however, is that of the superiority of TVol to existing prognostic variables, such as TSize, which has never truly been tested in PDAC.

In 1993, Sellner and Machacek (7) reported their analysis of a cohort of patients with mixed periampullary cancer, in a study that grouped together patients with lower common bile duct cancer, cancer of the ampulla of Vater and 49 patients with carcinoma of the pancreatic head. By using three dimensions to calculate TVol, as in the present study, the study demonstrated a negative association between survival time and TVol. This

\section{Survival between low and high volume}

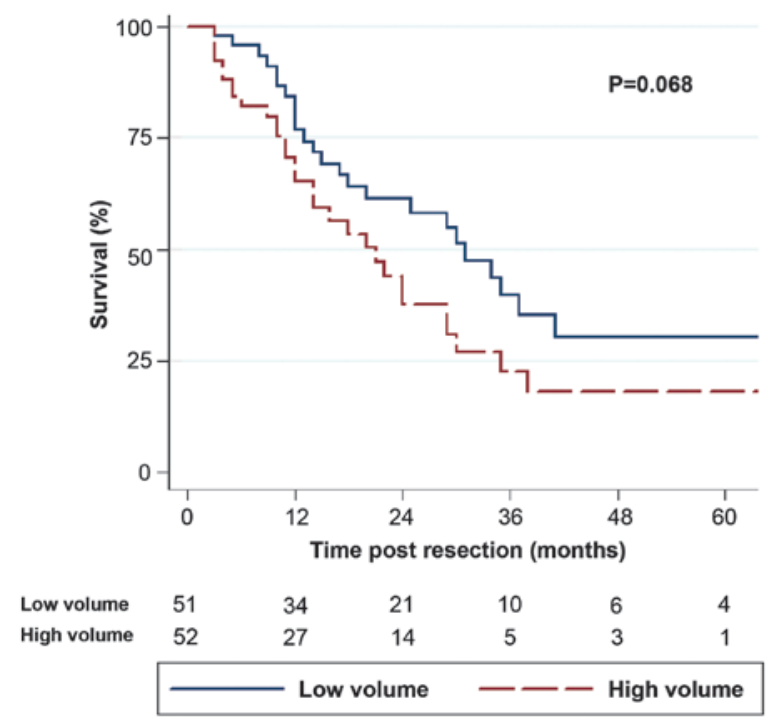

Figure 1. Survival curves in high and low tumour volume groups.

finding was reinforced by the present study and stands as the only other report of associations between clinical outcome and TVol in pancreatic cancer. Further discussion of the study by Sellner and Machacek (7) is limited by the fact that the three aforementioned and distinct malignancies were grouped for the purposes of further analysis. Given that these diseases are now known to harbour a specific natural history, and convey a differing prognosis, further information regarding PDAC TVol in isolation was unable to be deduced. Furthermore, no comparison with TSize was undertaken.

Despite previous studies showing significant correlations between outcome and TVol in a number of solid tumours (3-8), the present analysis failed to demonstrate the same degree of prognostic value beyond simple TSize. This is difficult to reconcile in the absence of previous studies with a specific focus on and adequate analysis of PDAC. It may be that the three-dimensional tumour burden (as calculated in the present study) does not convey the same prognostic value as it does in other malignancies.

Although previous studies have reported prognostic associations with TVol, it should also be noted that there exists disagreement in the literature, with prostate cancer being a good example. While the study of Chun et al (4) previously concluded that prostate $\mathrm{TVol}$ predicts prognosis, other studies have failed to find any correlation with outcome $(9,10)$. In a study of almost 900 men with localised prostate cancer and TVol data, Porten et al (9) conclude that 'there is no evidence that TVol is an independent predictor of prostate cancer outcome'. Additionally, Wolters et al found that although a computer-assisted determination of prostate TVol did correlate with existing markers of prognosis, volume itself failed to be a significant independent predictor of outcome following multivariate analysis (10). These findings are similar to those of the present study of post-resection PDAC outcome, whereby associations between existing prognostic markers (e.g., neural invasion) and TVol were observed (data not shown), but TVol was not shown to be an independent predictor of mortality. 
Table IV. Comparison between high and low TVol groups.

\begin{tabular}{|c|c|c|c|}
\hline Variable & Low TVol & High TVol & P-value \\
\hline Number of patients & 5 & 53 & \\
\hline Median age (range), years & $68(46-83)$ & $65.5(34-82)$ & 0.077 \\
\hline ASA grade & $2.0(1-4)$ & $2.0(1-3)$ & 0.469 \\
\hline Median surgical time (range), min & $390(290-620)$ & $430(290-720)$ & $<0.001$ \\
\hline Vascular resection, n (\%) & $20(39.2)$ & $34(65.4)$ & 0.007 \\
\hline Median intraoperative blood loss (range), $\mathrm{ml}$ & $500(90-2400)$ & $745(250-2360)$ & 0.007 \\
\hline Perioperative transfusion, $\mathrm{n}(\%)$ & $8(15.7)$ & $15(28.9)$ & 0.085 \\
\hline Complications, n (\%) & $16(31.4)$ & $20(38.5)$ & 0.537 \\
\hline Median hospital stay (range), days & $13(7-36)$ & $13(9-41)$ & 0.573 \\
\hline Median tumour size (range), $\mathrm{mm}$ & $30(15-45)$ & $45(27-100)$ & $<0.001$ \\
\hline Median tumour volume (range), $\mathrm{cm}^{3}$ & $5.37(0.6-11.8)$ & $20.9(11.5-138.2)$ & $<0.001$ \\
\hline \multicolumn{4}{|l|}{ Histological grade, n (\%) } \\
\hline Well & $2(3.9)$ & $3(5.8)$ & 0.280 \\
\hline Moderate & $33(64.7)$ & $35(67.3)$ & \\
\hline Poor & $16(31.4)$ & $11(2.1)$ & \\
\hline Undifferentiated & $3(5.8)$ & & \\
\hline Neural invasion, $\mathrm{n}(\%)$ & $36(70.6)$ & $40(76.9)$ & 0.306 \\
\hline Vascular invasion, $\mathrm{n}(\%)$ & $32(62.8)$ & $38(73.1)$ & 0.181 \\
\hline Positive margin, n (\%) & $18(35.3)$ & $18(34.6)$ & 0.553 \\
\hline Median lymph node yield (range), $\mathrm{n}$ & $17(4-65)$ & $17.5(6-43)$ & 0.436 \\
\hline Median lymph node-positive (range), $n$ & $37(72.6)$ & $36(69.2)$ & 0.439 \\
\hline 2-year survival, $\%$ & 41.2 & 26.9 & 0.068 \\
\hline
\end{tabular}

TVol, tumour volume; ASA, American Society of Anaesthesiologists.

Heterogeneity in the literature is further compounded by the various methods employed to calculate TVol; thus making comparisons between studies, even if focussed on the same tumour type, difficult. In the present study, the single centre pathology unit that was involved prospectively measured three tumour dimensions at the time of formal histopathological assessment. These values were collated retrospectively and the TVol was calculated using the formula for the volume of an ellipse. This method has successfully been applied to osteosarcoma (8) and nephrectomy specimens for renal cell carcinoma (5). In a subset of renal cell carcinoma patients, Jorns et al (5) showed that the risk of mortality was significantly higher in patients with an ellipsoidal TVol above the median compared with simple TSize above the median. Although not proving to be significant, a similar trend was observed in the present analysis of PDAC (Fig. 1) and suggests that the additional tumour dimensions can be useful in translating the true tumour burden, as it relates to mortality outcome.

A variety of methods have been reported in the literature to assess TVol and may explain certain disparities in the results between studies. Simple cuboidal (7) and ellipsoidal $(5,7,8)$ volume calculations based on macroscopic tumour dimensions have been supplemented by computer-assisted morphometric assessments, (10) magnetic resonance imaging volumetric reconstructions (6) and whole-body metabolic positron emission tomography volume imaging (3). The use of such imaging modalities to assess TVol and associations with outcome is an increasing trend that may ultimately lead to specific changes in management. Possessing the capacity to accurately predict who may or may not benefit from aggressive surgical intervention based on relatively simple indices, such as in vivo TVol, is an attractive proposition (2).

The method of calculating TVol would also theoretically benefit from inclusion of a correction factor based on the individual patient's body composition. It could be assumed that a 5-cm tumour in a 50-kg female represents a significantly larger tumour burden when compared to the same absolute TSize in a $100-\mathrm{kg}$ male. A simple method to normalise TVol for organ size has been employed previously in thyroid surgery and relies only on a simple calculation of body surface area (11). Minimal data regarding body composition (e.g., height and weight) was not available for the present analysis, but should be borne in mind for future studies. Although the resected pancreatic head dimensions and weight were available, these variables reflect more on the technical resection, rather than the patient's size.

Beyond independent TVol associations with mortality outcome, this study has revealed additional findings of significance. Univariate analysis showed that neural and vascular invasion were associated with a worse outcome, as 
was perioperative transfusion. These concepts have been highlighted previously (2) and the finding of neural invasion as an independent predictor of mortality following multivariate analysis supports its use as a prognostic and reported variable of significance.

It was also found that a higher TVol was associated with a closer pancreatic neck margin and a higher rate of formal vascular resection in the present study. In keeping with this, and as expected, a higher TVol is also correlated with longer surgical times and larger intraoperative blood losses. A longer surgery, vascular resection, closer pancreatic neck margins, higher intraoperative blood losses and perioperative transfusion are all known to be independently negative prognostic variables $(2,12-14)$. Multivariate analysis was therefore employed in the present study in an effort to control for the effect of these confounding factors on outcome when considering TVol and mortality in isolation.

A drawback of the present study was the absence of additional data regarding outcome and therapy. Knowledge of local recurrence, development of distant metastases, and the use of neo-adjuvant and adjuvant therapy would have been ideal in an attempt to control for all factors that affect outcome. This data was not available for the present analysis. It is also unknown if TVol more precisely predicts for recurrence, as opposed to mortality due to disease for instance.

In conclusion, the ability to accurately predict the true tumour burden and the impact this may have on the natural history of an individual patient's outcome is now more poignant than ever. Clinicians now work in a translational environment, and an individual patient's tumour genotype and phenotype are increasingly dictating management. Formal assessment of TVol represents additional information regarding tumour phenotype that should not be ignored, and in time, may be shown to be clearly superior to simple TSize, which is so highly emphasised in today's staging systems. The next challenge lies in identifying the best method to employ to extract this valuable prognostic information.

\section{References}

1. AJCC: Staging Resources. 2013; http://www.cancerstaging.org/. Accessed July 1, 2014.
2. Garcea G, Dennison AR, Pattenden CJ, Neal CP, Sutton $\mathrm{CD}$ and Berry DP: Survival following curative resection for pancreatic ductal adenocarcinoma. A systematic review of the literature. JOP 9: 99-132, 2008.

3. Oh JR, Seo JH, Chong A, Min JJ, Song HC, Kim YC and Bom HS: Whole-body metabolic tumour volume of $18 \mathrm{~F}-\mathrm{FDG}$ PET/CT improves the prediction of prognosis in small cell lung cancer. Eur J Nucl Med Mol Imaging 39: 925-935, 2012.

4. Chun FK, Briganti A, Jeldres C, Gallina A, Erbersdobler A, Schlomm T, Walz J, Eichelberg C, Salomon G, Haese A, et al: Tumour volume and high grade tumour volume are the best predictors of pathologic stage and biochemical recurrence after radical prostatectomy. Eur J Cancer 43: 536-543, 2007.

5. Jorns J, Thiel DD, Lohse CM, Williams A, Arnold ML, Cheville JC, Leibovich BC and Parker AS: Three-dimensional tumour volume and cancer-specific survival for patients undergoing nephrectomy to treat pT1 clear-cell renal cell carcinoma. BJU Int 110: 956-960, 2012.

6. Chew MH, Khoo JBK, Chong VFH, Tai BC, Soo KC and Lim DT: Significance of tumour volume measurements in tongue cancer: A novel role in staging. ANZ J Surg 77: 632-637, 2007.

7. Sellner F and Machacek E: The importance of tumour volume in the prognosis of radically treated periampullary carcinomas. Eur J Surg 159: 95-100, 1993.

8. Munajat I, Zulmi W, Norazman MZ and Wan Faisham WI: Tumour volume and lung metastasis in patients with osteosarcoma. J Orthop Surg (Hong Kong) 16: 182-185, 2008.

9. Porten SP, Cooperberg MR and Carroll PR: The independent value of tumour volume in a contemporary cohort of men treated with radical prostatectomy for clinically localized disease. BJU Int 105: 472-475, 2010.

10. Wolters T, Roobol MJ, van Leeuwen PJ, van den Bergh RC, Hoedemaeker RF, van Leenders GJ, Schröder FH and van der Kwast TH: Should pathologists routinely report prostate tumour volume? The prognostic value of tumour volume in prostate cancer. Eur Urol 57: 821-829, 2010.

11. Moon HG, Jung EJ, Park ST, Jung TS, Jeong CY, Ju YT, Lee YJ, Hong SC, Choi SK and Ha WS: Thyrotropin level and thyroid volume for prediction of hypothyroidism following hemithyroidectomy in an Asian patient cohort. World J Surg 32: 2503-2508, 2008.

12. Chang DK, Johns AL, Merrett ND, Gill AJ, Colvin EK, Scarlett CJ, Nguyen NQ, Leong RW, Cosman PH, Kelly MI, et al: Margin clearance and outcome in resected pancreatic cancer. J Clin Oncol 27: 2855-2862, 2009.

13. Fatima J, Schnelldorfer T, Barton J, Wood CM, Wiste HJ, Smyrk TC, Zhang L, Sarr MG, Nagorney DM and Farnell MB: Pancreatoduodenectomy for ductal adenocarcinoma: Implications of positive margin on survival. Arch Surg 145: 167-172, 2010.

14. Fukuda S, Oussoultzoglou E, Bachellier P, Rosso E, Nakano H, Audet $\mathrm{M}$ and Jaeck D: Significance of the depth of portal vein wall invasion after curative resection for pancreatic adenocarcinoma. Arch Surg 142: 172-180, 2007. 